\title{
Contributions towards Data driven Deep Learning methods to predict Steady State Fluid Flow in mechanical Heart Valves
}

\author{
Jan Oldenburg*, Institute for ImplantTechnology and Biomaterials e.V., Rostock-Warnemünde, Germany, \\ jan.oldenburg@uni-rostock.de \\ Julian Renkewitz, Institute for Biomedical Engineering, Rostock University Medical Center, Rostock, Germany, \\ julian.renkewitz@uni-rostock.de \\ Klaus-Peter Schmitz, Institute for ImplantTechnology and Biomaterials e.V., Rostock-Warnemünde, Germany, klaus- \\ peter.schmitz@uni-rostock.de \\ Michael Stiehm, Institute for ImplantTechnology and Biomaterials e.V., Rostock-Warnemünde, Germany, \\ michael.stiehm@uni-rostock.de
}

\section{Introduction:}

It is commonly accepted that hemodynamic situation is related with cardiovascular diseases as well as clinical postprocedural outcome. In particular, aortic valve stenosis and insufficiency are associated with high shear flow and increased pressure loss. Furthermore, regurgitation, high shear stress and regions of stagnant blood flow are presumed to have an impact on clinical result. Therefore, flow field assessment to characterize the hemodynamic situation is necessary for device evaluation and further design optimization.

\section{Methods:}

In-vitro as well as in-silico fluid mechanics methods can be used to investigate the flow through prostheses. In-silico solutions are based on mathematical equitation's which need to be solved numerically (Computational Fluid Dynamics - CFD). Fundamentally, the flow is physically described by Navier-Stokes. CFD often requires high computational cost resulting in long computation time. Techniques based on deep-learning are under research to overcome this problem. In this study, we applied a deep-learning strategy to estimate fluid flows during peak systolic steady-state blood flows through mechanical aortic valves with varying opening angles in randomly generated aortic root geometries. We used a data driven approach by running 3,500 two dimensional simulations (CFD). The simulation data serves as training data in a supervised deep learning framework based on convolutional neural networks analogous to the U-net architecture.

\section{Results:}

We were able to successfully train the neural network using the supervised data driven approach. The results showing that it is feasible to use a neural network to estimate physiological flow fields in the vicinity of prosthetic heart valves (Validation error below 0.06 ), by only giving geometry data (Image) into the Network. The neural network generates flow field prediction in real time, which is more than 4,000 times faster compared to CFD simulation.

\section{Conclusion:}

Accordingly, there is tremendous potential in the use of Al-based approaches predicting blood flows through heart valves on the basis of geometry data, especially in applications where fast fluid mechanic predictions are desired. 


\title{
Generation of cellularized fibrin-based bioartificial blood vessels with physiological elastic properties by transluminal matrix compression
}

\author{
Florian Helms ${ }^{1,2}$, Axel Haverich ${ }^{1,2}$, Ulrike Böer ${ }^{1,2}$, Mathias Wilhelmi ${ }^{1,3}$ \\ ${ }^{1}$ Lower Saxony Center for Biomedical Engineering, Implant Research and Development, Hannover, Germany \\ ${ }^{2}$ Department of Cardiothoracic, Transplant and Vascular Surgery, Hannover Medical School, Hannover, Germany \\ ${ }^{3}$ Clinic for Vascular and Endovascular Surgery, St. Bernward Hospital, Hildesheim, Germany \\ Corresponding author: Florian Helms: helms.florian@mh-hannover.de
}

\section{Introduction}

Small-diameter vessel diseases cause by far the largest share of morbidity and mortality on a population level worldwide. Meanwhile, currently used alloplastic graft materials for bypassing diseased vessels are still prone to complications by insufficient biocompatibility and lack of physiological elastic properties, which ultimately leads to graft stenoses and occlusions. Fibrin-based tissue-engineered bioartificial blood vessels represent a promising alternative to overcome these limitations. However, broad clinical application of fibrin-based grafts is still prohibited by insufficient biomechanical stability and stiffness of fibrin matrices until today.

\section{Methods}

As an attempt to optimize strength and elasticity of fibrin matrices for vascular tissue engineering applications, we developed a method for transluminal compression of small-diameter fibrin-based blood vessels: After initial polymerization of high-concentration fibrin matrices in a vascular mold, vessels were compressed by inflating a standard angioplasty balloon (6 F, $8 \mathrm{~mm}$ x $200 \mathrm{~mm}$ ) inserted into the lumen of the bioartificial graft. Vessels compacted with different filling pressures were compared for ultimate mechanical strength, elastic and structural properties and cellularization capacity.

\section{Results}

Transluminal compression facilitated rapid production of homogenous vessels with a length of $10 \mathrm{~cm}$ and a diameter of approximately $7.5 \mathrm{~mm}$. Scanning electron microscopy revealed increased fibrin network density after compression. Compared to non-compressed controls, compacted fibrin vessels showed superior maximal burst pressure $(199.8 \mathrm{mmHg}$ vs. $94.0 \mathrm{mmHg}$ ), physiological elastic properties similar to the elastic behavior of natural arteries and higher luminal endothelial cell coverage $(98.6 \%$ vs. $34.6 \%)$.

\section{Conclusion}

In conclusion, transluminal compression is a simple and reproducible technique to generate highly compacted fibrin matrices and enhance biomechanical properties of fibrin-based bioartificial blood vessels while preserving excellent cellularization capacities. With this, it represents a useful tool on the way towards clinical application of bioengineered vessels. 


\section{TAVI-Mimic for testing of heart valve leaflet materials in physiologi- cal loading situations}

Joschka Finck, Institute for Biomedical Engineering, University Medical Center Rostock, Germany, joschka.finck@uni-rostock.de

Jan Oldenburg, Institute for ImplantTechnology and Biomaterials e.V., Rostock-Warnemünde, Germany, Thomas Kuske, Institute for ImplantTechnology and Biomaterials e.V., Rostock-Warnemünde, Germany, Niels Grabow, Institute for Biomedical Engineering, University Medical Center Rostock, Germany

Klaus-Peter Schmitz, Institute for Implant Technology and Biomaterials e.V., Rostock-Warnemünde, Germany, | Institute for Biomedical Engineering, University Medical Center Rostock, Germany

Stefan Siewert, Institute for ImplantTechnology and Biomaterials e.V., Rostock-Warnemünde, Germany, Stefan Oschatz, Institute for Biomedical Engineering, University Medical Center Rostock, Germany

Michael Stiehm, Institute for ImplantTechnology and Biomaterials e.V., Rostock-Warnemünde, Germany, Sebastian Kaule, Institute for ImplantTechnology and Biomaterials e.V., Rostock-Warnemünde, Germany

\section{Introduction}

The loading situation of the aortic valve is complex, complicating the identification of innovative approaches for heart valve leaflet materials, e.g. transcatheter aortic valve implantation (TAVI). Materials engineering experiments allow for screening of materials but especially for durability testing, the consideration of physiological loads is vital/critical for the suitability-assessment of innovative leaflet materials.

For this reason, a framework structure for the testing of leaflet materials in physiological loading (TAVI-Mimic) was developed. The exemplary use case for the TAVI-Mimic was a test for calcification propensity of pericardium during durability testing.

\section{Methods}

The TAVI-Mimic was designed as a four-parted frame, based on previous work of our group. The leaflet material can be attached between inner and outer shells without sewing. In a second step, the TAVI-Mimic was optimized regarding radial load-deformation in comparison to a commercial TAVI by means of finite element analysis (FEA) and hydrodynamic characterization in a pulse duplicator system. Mechanical properties dependent on water update of different materials for 3D-printing of the TAVI-Mimic were investigated.

After optimization, TAVI-Mimics were equipped with glutaraldehyde-fixated pericardial tissue and prototypes were calcified by using a heart valve durability tester and a metastable calcification-liquid, developed in earlier studies.

\section{Results}

The development of the TAVI-Mimic using FEA and experiments was successful, leading to a radial load dependent deformation of $0.6 \mathrm{~mm}$ which correlates with commercial TAVI. Two methacrylic photopolymers were identified for 3D-printing of the TAVI-Mimic and prototypes attached with pericardial tissue were manufactured.

Pericardium TAVI-Mimics were calcified in vitro for one week and an average calciumphosphate precipitate of 0.34$0.54 \mathrm{mg} / \mathrm{cm}^{2}$ was measured.

\section{Conclusion}

The optimization of the TAVR-Mimic led to an improved load-dependent behavior compared to a commercial prosthesis while testing. The calcification method, combining the TAVI-Mimic, the metastable calcification solution and the durability tester enabled a successfully calcification of pericardial tissue, approaching the in vivo situation. 


\section{Investigation of physico-chemical properties of TSPCU nonwoven for application in prosthetic venous valves}

Julia Schubert, Institute for Biomedical Engineering, Rostock University Medical Center, Rostock, Germany, julia.schubert@uni-rostock.de

Daniela Arbeiter, Institute for Biomedical Engineering, Rostock University Medical Center, Rostock, Germany, daniela.arbeiter@uni-rostock.de

Andreas Götz, Institute for Biomedical Engineering, Rostock University Medical Center, Rostock, Germany, andreas.goetz@uni-rostock.de

Kerstin Lebahn, Institute for Biomedical Engineering, Rostock University Medical Center, Rostock, Germany, kerstin.schuemann@uni-rostock.de

Wolfram Schmidt, Institute for Biomedical Engineering, Rostock University Medical Center, Rostock, Germany, wolfram.schmidt@uni-rostock.de

Niels Grabow, Institute for Biomedical Engineering, Rostock University Medical Center, Rostock, Germany, niels.grabow@uni-rostock.de

Sabine Illner, Institute for Biomedical Engineering, Rostock University Medical Center, Rostock, Germany, sabine.illner@uni-rostock.de

\section{Introduction}

Electrospinning has become an emerging method to produce nonwovens for medical polymer-based implants, such as prosthetic valves or covered scaffolds. In this study, nonwovens for prosthetic venous valves are investigated regarding their morphology and mechanics in physiological medium.

\section{Methods}

Spinning molds were developed based on previous venous valve leaflet designs, 3D printed in different sizes and covered with electrospun nonwovens. For several weeks samples were stored in a physiological $0.9 \%$ saline at $37^{\circ} \mathrm{C}$ to investigate the influence of fiber rearrangement and swelling in medium. Two different nonwovens made of thermoplastic silicone-based polycarbonaturethane (TSPCU) with different material hardness (TSPCU I - 80A and TSPCU II - 55D) were compared with respect to their morphological and mechanical properties depending on their storage time in saline.

\section{Results}

Tensile test results show that storage in medium has a relevant influence on the mechanical properties of the TSPCU nonwovens. Compared to dry samples measured in air, Young's modulus and tensile strength of dry samples measured in $0.9 \%$ saline at $37^{\circ} \mathrm{C}$ are significantly decreased by at least $60 \%$ and $35 \%$, respectively. Comparing mechanical behavior of dry samples and 4 weeks medium-stored samples, all measured in medium, an increase in Young's modulus and tensile strength of at least $48 \%$ and $28 \%$, was observed. SEM images for TSPCU I and II show substantial increased fiber diameter after 8 days stored in medium. After detaching the valve leaflet nonwovens from the molds, shrinkage of the material of approximately $12 \%$ was detected. A suitable valve size could be identified for joining with the stent structure into an interventional prosthetic venous valve.

\section{Conclusion}

The results demonstrate the influence of storage conditions on the morphological and mechanical properties of electrospun nonwovens made of TSPCU. For development and dimensioning of venous valve leaflets, this change in mechanical behavior and possible shrinkage of the material has to be considered in further investigations. 


\section{Cardiovascular catheter stiffness - a static measurement approach}

Christoph Brandt-Wunderlich, Institute for ImplantTechnology and Biomaterials e.V., Rostock-Warnemünde, Germany, e-Mail: christoph.brandt-wunderlich@uni-rostock.de

Niels Grabow, Institute for Biomedical Engineering, Rostock University Medical Center, Rostock-Warnemünde, Germany, e-Mail: niels.grabow@uni-rostock.de

Klaus-Peter Schmitz, Institute for ImplantTechnology and Biomaterials e.V and Institute for Biomedical Engineering, Rostock University Medical Center, Rostock-Warnemünde, Germany, e-Mail: klaus-peter.schmitz@uni-rostock.de Stefan Siewert, Institute for ImplantTechnology and Biomaterials e.V, Rostock-Warnemünde, Germany, e-Mail: stefan.siewert@uni-rostock.de

Wolfram Schmidt, Institute for Biomedical Engineering, Rostock University Medical Center, Rostock-Warnemünde, Germany, e-Mail: wolfram.schmidt@uni-rostock.de

\section{Introduction}

Catheters are widely used for therapeutic and diagnostic purposes in various medical applications. Typically, catheters are introduced minimally invasive and then advanced to the target location. Therefore, the handling properties of the used device significantly affect the success of the clinical procedure. Within the current study a static measurement approach for catheter stiffness analysis is presented.

\section{Methods}

Measurement accuracy and uncertainty were assessed with respect to the free catheter length and sample stiffness. Three test samples with a length of $120 \mathrm{~mm}$ were cut from the distal and proximal region of commercial available coronary balloon catheters, respectively. Using a custom made automated test setup, the test samples were deflected at a certain clamping length $(10 \mathrm{~mm}$ to $50 \mathrm{~mm})$, while measuring the reaction force. Measurements were performed three times at five positions in circumferential direction. Bending stiffness was calculated directly from the measured force, deflection and clamping length considering the test setup compliance.

\section{Results}

Measured bending stiffness ranges from $629 \pm 31 \mathrm{Nmm}^{2}$ to $733 \pm 58 \mathrm{Nmm}^{2}$ for the proximal samples and from $30 \pm 5 \mathrm{Nmm}^{2}$ to $98 \pm 30 \mathrm{Nmm}^{2}$ for the distal samples, respectively. Bending stiffness varied depending on the free catheter length and the reaction force measured. The maximum reaction force decreased with increasing free catheter length leading to a higher measurement uncertainty. However, when considering the same free catheter length quantitative results were similar for proximal and distal samples, respectively.

\section{Conclusion}

The quantitative test results confirm lower bending stiffness in the distal catheter section compared with the proximal section. For direct comparison of the test results the same free catheter length should be used. Furthermore, a minimized free catheter length results in higher reaction forces and therefore reduces measurement uncertainties. The presented custom made test setup is suitable for investigations of bending properties of catheters and other medical devices, such as stents. 